\title{
Editorial
}

\section{The epidemiological situation of COVID-19 in the Kyrgyz Republic}

The official registration of an outbreak of coronavirus infections in the Kyrgyz Republic began on March 12, 2020. In the weekly dynamics, the peak number of cases of coronavirus infection occurred in the fourth week of the development of the epidemic process (Fig. 1).

The maximum number of cases of coronavirus infection was 79 (tested positive cases, including asymptomatic cases and cases with clinical manifestations) as of April 12, 2020. According to the forecast by the WHO methodology, the peak incidence of COVID-19 should be at the eighth week of the development of the epidemic process, amounting to 92 cases. Starting from the fifth week, the number of cases of COVID-19 tends to decrease gradually and the mathematical model for predicting the incidence of COVID-19 in the Kyrgyz Republic shows that by the end of May 2020, $97 \%$ of the total number of infected with coronavirus will be detected.

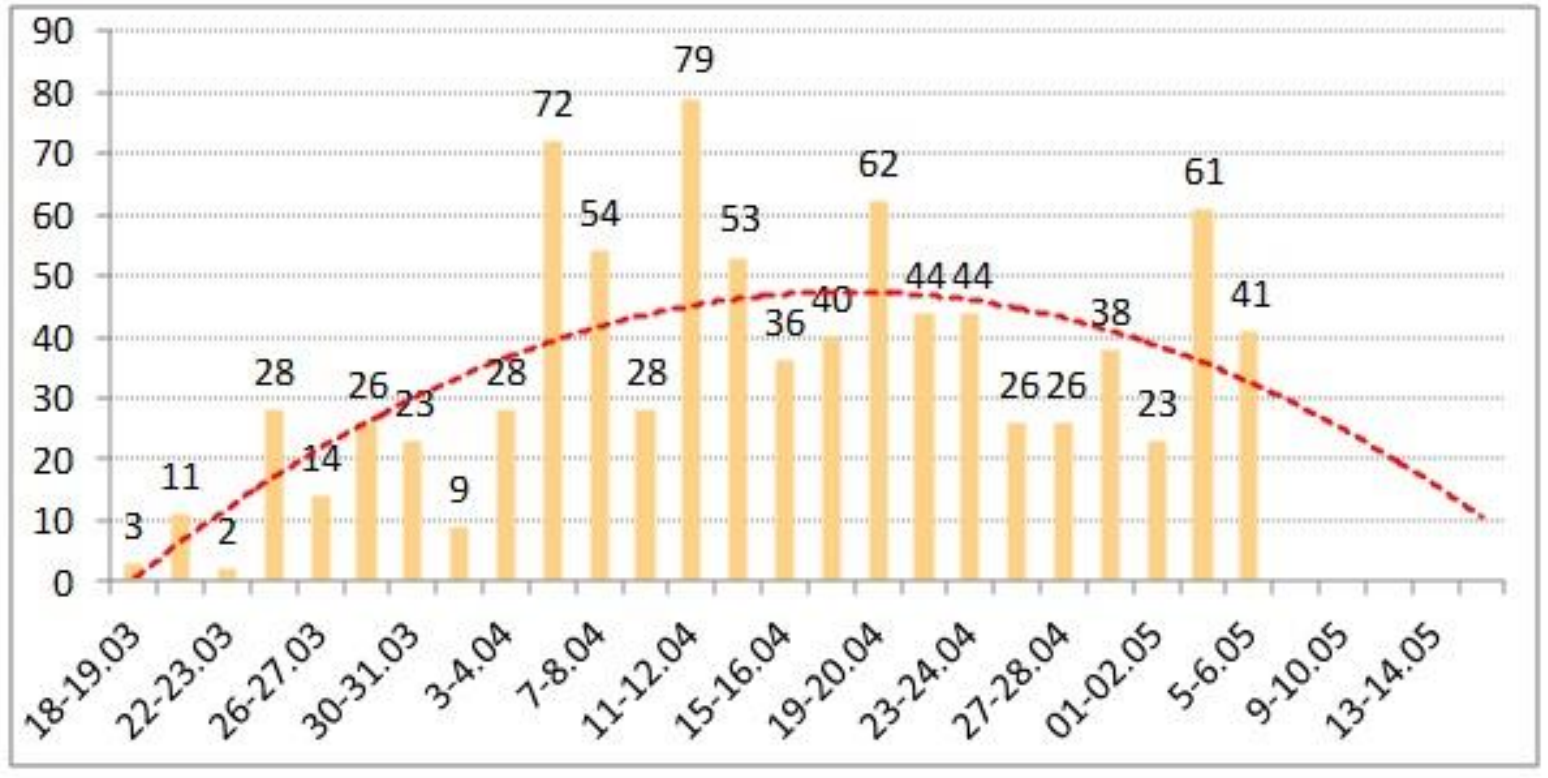

Figure 1. Dynamics of the number of cases of COVID-19 in the Kyrgyz Republic (March 19 to May 6, 2020, the absolute number of new cases is $\mathbf{8 7 1}$ )

On May 6, 871 confirmed cases of COVID-19 infection were recorded. Of the registered positive cases - 465 women, 406 - men, 614 patients were discharged, which is $70.5 \%$. Overall, 245 patients receive treatment: of these, $7(2.9 \%)$ are severe, $60(24.5 \%)$ are moderate and $178(72.2 \%)$ are mild and asymptomatic cases. At present, $67.8 \%$ of the detected positive cases are asymptomatic, who are hospitalized for epidemiological reasons, to prevent the further spread of infection; treatment in these cases is symptomatic if necessary. Complications are as following: bilateral pneumonia, multiple organ failure syndrome, cerebral edema. Concomitant diseases are cirrhosis of the liver with portal hypertension, exudative pleurisy. In total, 12 people died with a diagnosis of coronavirus infection in Kyrgyzstan, the mortality rate is $1.4 \%$.

Address for Correspondence: Vera S. Toigombaeva, Department of General and Clinical Epidemiology, Kyrgyz State Medical Academy, Bishkek, Kyrgyzstan, Email: vera2808@inbox.ru

Received: 07.05.2020 Accepted: 09.05.2020 
Registration of COVID-19 was observed in all regions except the Talas oblast. The largest number of registered cases of COVID-19 is in Bishkek, as the bulk of visitors from disadvantaged countries arrive through the Manas airport and the Ak-Zhol border post. All arrivals were accommodated in observation facilities located in the city. They were quarantined for 14 days with taking material for laboratory analysis upon arrival and on the 12th day of observation (*Medical observation during a certain period of time of healthy people isolated in a special room who might have contact with sick quarantine diseases. The observation is guarded 24 hours per day by law enforcement officials to maintain the order provided for those in the observation.). Observation facilities are located in all major cities of the republic.

Of all the cases of COVID-19 in the republic (871), the southern regions account for $54 \%$, Bishkek $-29.3 \%$ and Osh $-12 \%$. By region, the highest intensive indicators (Intensive indicators were calculated for the actual population of each region per 100,000 population) are in Osh (36.970 / 0000), Naryn Oblast (35.770 / 0000) and Bishkek (24.570 / 0000), followed by Osh (12.570 / 0000), Jalal-Abad (10.570 / 0000), Chuy (7.870 / 0000), Batken (3.470 / 0000) and Issyk-Kul (2.070 / 0000) regions (Fig. 2).

\section{Epidemiologic situation of COVID-19 in KR by 06.05 .2020 (abs.number per 100,000 population)}

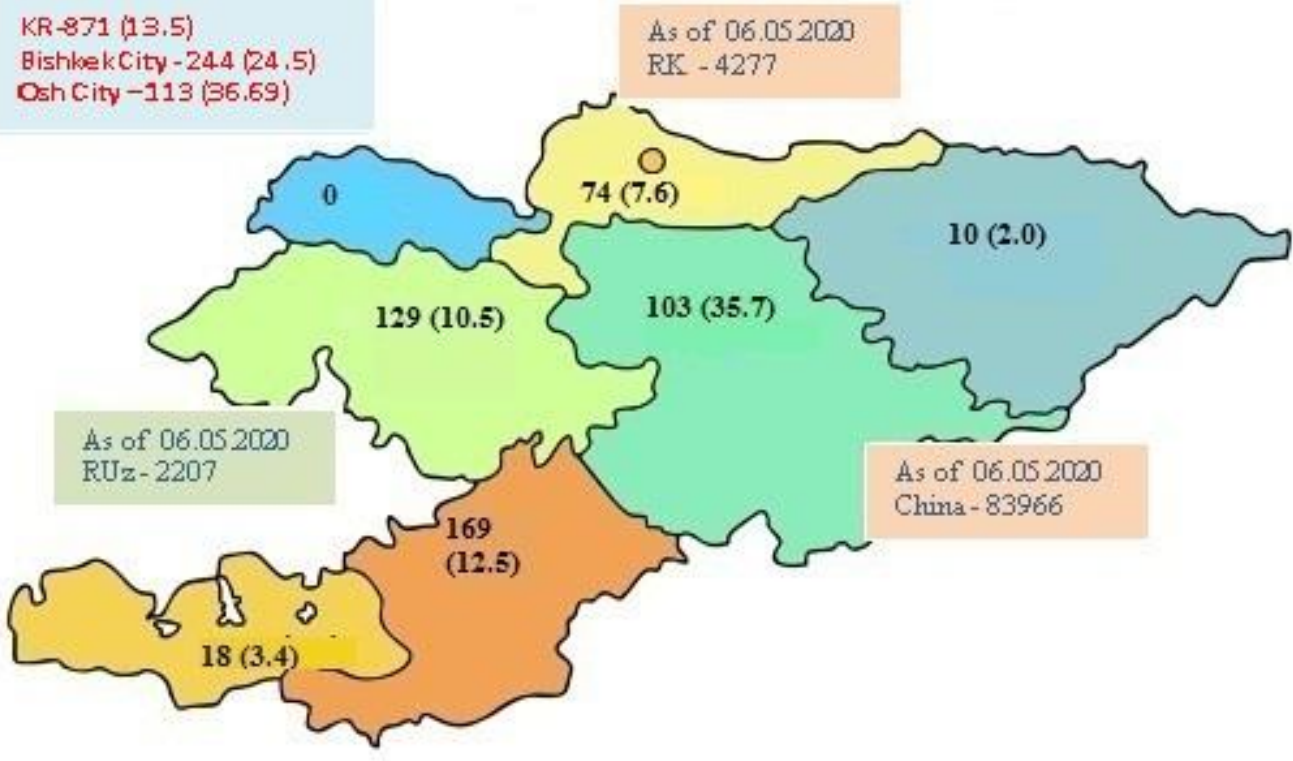

Figure 2. Distribution of COVID-19 in the regions of the Kyrgyz Republic on 05/06/2020 (absolute number and per 100 thousand of the population)

KR - Kyrgyz Republic, RK - Republic of Kazakhstan, RUz - Republic of Uzbekistan

Of particular concern is the increase in the number of cases of infection of medical workers reaching $25.4 \%$ of the total. Over the entire period of COVID-19 distribution, a total of 221 cases of infection of medical workers were recorded, of whom 154 were discharged from hospitals with recovery. The largest number of cases of infection of medical workers was noted in
Bishkek (85), Osh city (38), Osh (33) and Naryn (27) regions (oblasts). Registration of COVID-19 among health workers began at the end of the 2 nd week of the development of the epidemic process, its dynamics had a pronounced upward trend, and at present, rising by $282 \%$ (Fig. 3). 


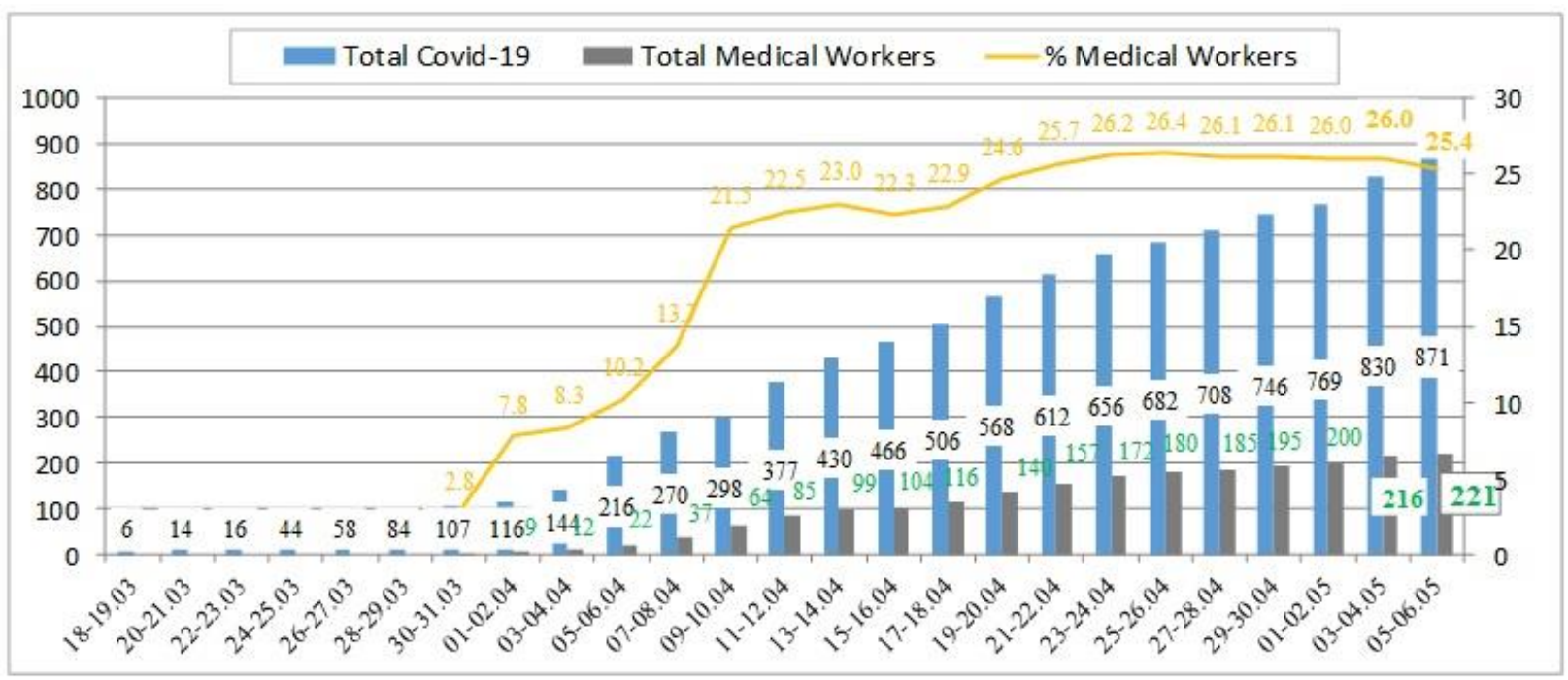

Figure 3. The dynamics of the number of cases of COVID-19 among the population and medical workers

High rates of infection among medical workers are associated with the fact that they are in direct contact with patients, with citizens suspicious of the presence of coronavirus, and with contacts among the population. We assume that with full provision of personal protective equipment (PPE) and acquired skills in their use, the incidence rate of workers in healthcare organizations will decrease. Currently (May 7, only 4 cases) there is a decrease in the number of infections among medical workers.

In total, for the analyzed period, 59490 laboratory tests were conducted in the republic to identify COVID-19. Currently there are 675 people on observation (quarantine, see above*). These are people who are being monitored on the occasion of contact with COVID-19 and who arrived from disadvantaged countries. According to the algorithm of action in relation to persons under observation, they should be examined 2 times for coronavirus and, based on the results of 2 negative tests and the absence of the clinical manifestations, are discharged.

The state of emergency was introduced from March 18 and currently remains until May 10 inclusive. Currently, in connection with the ongoing registration of cases, the emergency mode is maintained, and group and public events are limited. Educational institutions and schools operate ONLINE, public transport does not work, you can only use a taxi, and permission has been given to the activities of some enterprises in the service sector. To comply with the state of emergency, a total of 535 checkpoints were created in the republic, including 24 sanitary-quarantine checkpoints. While maintaining the downward trend in the registration of COVID-19, it is planned that the state of emergency will be removed from May 11, 2020 in the administrative territories of Osh, Jalal-Abad and At-Bashi district of the Naryn region, to remove the state of emergency in Bishkek until May 31, 2020 and maintained emergency mode throughout the Kyrgyz Republic. The main factors that can change the dynamics of the incidence rate are: state of emergency and emergency modes, provision of PPE for medical workers and their proper use, adherence to hand hygiene rules by the population, wearing masks, distancing. On the part of employers strict compliance with all COVID-19 prevention requirements at facilities.

${ }^{1}$ Vera S. Toigombaeva, ${ }^{2}$ Zuridin Sh. Nurmatov

${ }^{1}$ Department of General and Clinical Epidemiology, Kyrgyz State Medical Academy, Bishkek, Kyrgyzstan

${ }^{2}$ Republic Scientific-Practical Center on Virus Infections, SPA "Preventive Medicine" of the Ministry of Health of Kyrgyz Republic, Bishkek, Kyrgyzstan

Peer-review: Internal

Conflict of interest: None to declare

Authorship: V.S.T and Z.Sh.N. are equally contributed to preparation of article

Acknowledgement and Funding: None to declare 


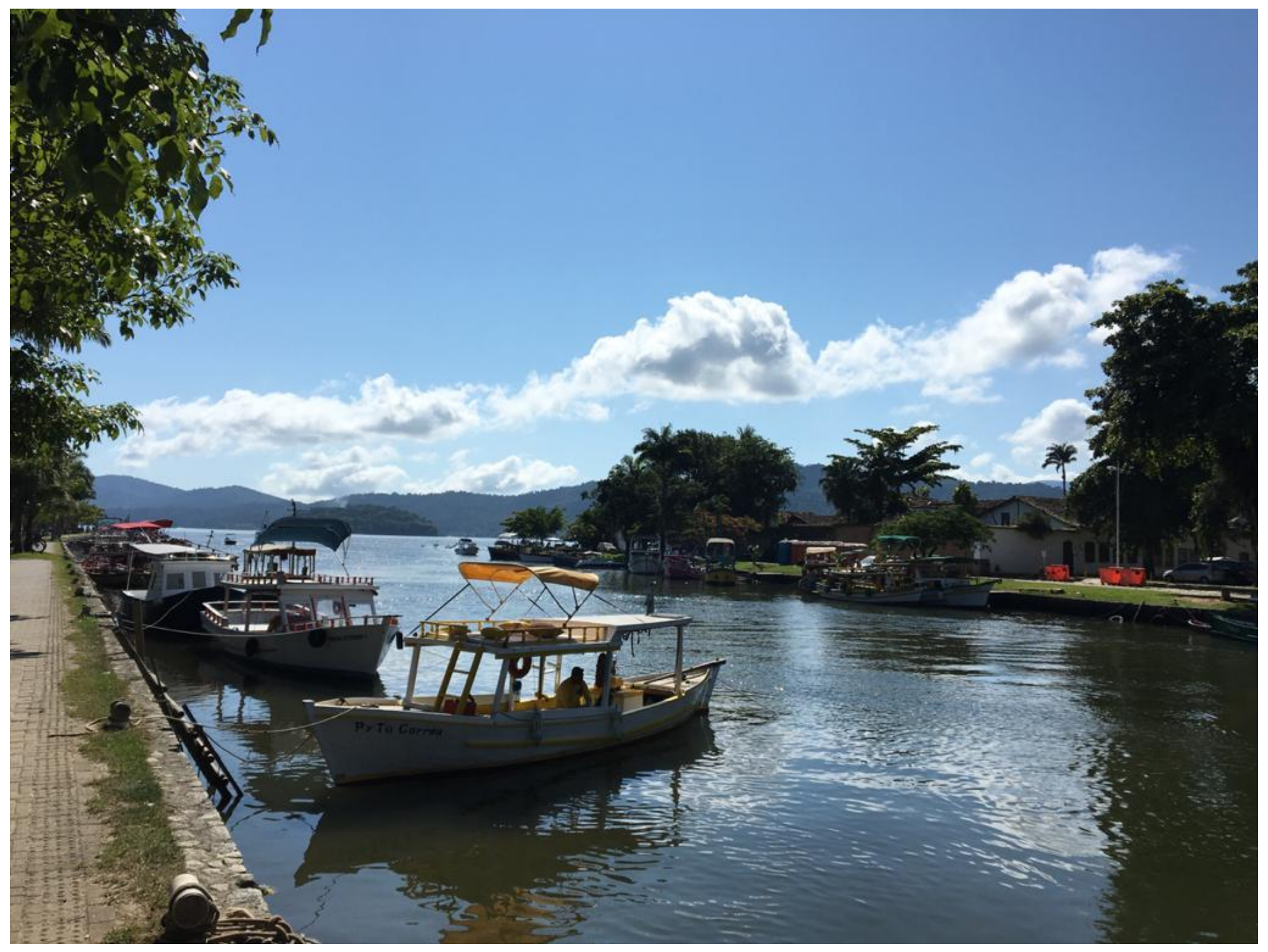

Pre-quarantine Paraty, State of Rio de Janeiro, Brazil . Andrea Rocha de Lorenzo, Rio de Janeiro, Brazil. 